\title{
A new approach to BVPs with state-dependent impulses
}

\author{
Irena Rachůnková* and Jan Tomeček
}

"Correspondence:
irena.rachunkova@upol.cz
Department of Mathematical
Analysis and Applications of
Mathematics, Faculty of Science,
Palacký University, 17. listopadu 12,
Olomouc, 771 46, Czech Republic

Olomouc, 771 46, Czech Republic

\begin{abstract}
The paper deals with the second-order Dirichlet boundary value problem with one state-dependent impulse

$$
\begin{aligned}
& z^{\prime \prime}(t)=f(t, z(t)) \quad \text { for a.e. } t \in[0, T], \\
& z^{\prime}(\tau+)-z^{\prime}(\tau-)=l(z(\tau)), \quad \tau=\gamma(z(\tau)), \\
& z(0)=0, \quad z(T)=0 .
\end{aligned}
$$

Proofs of the main results contain a new approach to boundary value problems with state-dependent impulses which is based on a transformation to a fixed point problem of an appropriate operator in the space $C^{1}([0, T]) \times C^{1}([0, T])$. Sufficient conditions for the existence of solutions to the problem are given here. The presented approach can be extended to more impulses and to other boundary conditions.
\end{abstract} MSC: 34B37; 34B15

Keywords: impulsive differential equation; state-dependent impulses; Dirichlet problem; second-order ODE

\section{Introduction}

Differential equations involving impulse effects appear as a natural description of observed evolution phenomena of several real world problems. We refer to the monographs [1-3].

Most papers in the literature on impulsive boundary value problems concern the case with fixed moments of impulsive effects. Papers dealing with state-dependent impulses, called also impulses at variable times, focus their attention on initial value problems or periodic problems. Such papers investigate the existence, stability or asymptotic properties of solutions of initial value problems [4-8] or solvability of autonomous periodic problems $[9,10]$ and nonautonomous ones [11-15]. We can also find papers investigating other boundary value problems with state-dependent impulses through some initial value problems for multi-valued maps [16, 17].

In this paper we provide a new approach to boundary value problems with statedependent impulses based on a construction of proper sets and operators and the topological degree arguments. Unlike previous existing results, our approach enables us to find simple existence conditions for data functions and it can be used for other regular (and also singular) problems. We demonstrate it on the second-order Dirichlet boundary value 
problem with one state-dependent impulse

$$
\begin{aligned}
& z^{\prime \prime}(t)=f(t, z(t)) \quad \text { for a.e. } t \in[0, T], \\
& z^{\prime}(\tau+)-z^{\prime}(\tau-)=I(z(\tau)), \quad \tau=\gamma(z(\tau)), \\
& z(0)=0, \quad z(T)=0,
\end{aligned}
$$

where we assume

$$
\begin{aligned}
& f \in \operatorname{Car}([0, T] \times \mathbb{R}), \quad I \in C(\mathbb{R}), \\
& \left\{\begin{array}{l}
\text { there exists } h \in \operatorname{Car}([0, T] \times[0, \infty)) \text { such that } \\
h(t, \cdot) \text { is nondecreasing for a.e. } t \in[0, T] \text { and } \\
f(t, x) \mid \leq h(t,|x|) \text { for a.e. } t \in[0, T] \text { and all } x \in \mathbb{R},
\end{array}\right. \\
& \left\{\begin{array}{l}
\text { there exists } J \in C([0, T]) \text { nondecreasing } \\
\text { and such that }|I(x)| \leq J(|x|) \text { for } x \in \mathbb{R},
\end{array}\right. \\
& \exists K>0: \frac{1}{K}\left[\int_{0}^{T} h(s, K+T J(K)) \mathrm{d} s+J(K)\right]<\min \left\{1, \frac{1}{T}\right\}
\end{aligned}
$$

and

$$
\left\{\begin{array}{l}
\gamma \in C^{1}\left(\left[-K_{1}, K_{1}\right]\right), \\
0<\gamma(x)<T, \quad\left|\gamma^{\prime}(x)\right|<\frac{T}{K_{1}} \quad \text { for }|x| \leq K_{1}, \\
\text { where } K_{1}=K+T J(K), \quad K \text { is from }(7) .
\end{array}\right.
$$

Under assumptions (4)-(8), we prove the solvability of problem (1)-(3). In particular, we transform problem (1)-(3) to a fixed point problem for a proper operator in the space $C^{1}([0, T]) \times C^{1}([0, T])$. This approach can be also used for other types of boundary conditions and it can be easily extended to more impulses.

Here, we denote by $C(J)$ the set of all continuous functions on the interval $J$, by $C^{1}(J)$ the set of all functions having continuous derivatives on the interval $J$ and by $L^{1}(J)$ the set of all Lebesgue integrable functions on $J$. For a compact interval $J$, we consider the linear space of functions from $C(J)$ or $C^{1}(J)$ equipped, respectively, with the norms

$$
\|x\|_{\infty}=\max _{t \in J}|x(t)|, \quad\|x\|_{1}=\|x\|_{\infty}+\left\|x^{\prime}\right\|_{\infty} .
$$

In this paper we work with the linear space $C^{1}([0, T]) \times C^{1}([0, T])$, where $T>0$, equipped with the norm

$$
\|(u, v)\|=\|u\|_{1}+\|v\|_{1} \quad \text { for }(u, v) \in C^{1}([0, T]) \times C^{1}([0, T]) .
$$

It is well-known that the mentioned normed spaces are Banach spaces. Recall that for $\mathcal{A} \subset \mathbb{R}$, a function $f:[a, b] \times \mathcal{A} \rightarrow \mathbb{R}$ satisfies the Carathéodory conditions on $[a, b] \times \mathcal{A}$ (we write $f \in \operatorname{Car}([a, b] \times \mathcal{A})$ ) if

- $f(\cdot, x):[a, b] \rightarrow \mathbb{R}$ is measurable for all $x \in \mathcal{A}$,

- $f(t, \cdot): \mathcal{A} \rightarrow \mathbb{R}$ is continuous for a.e. $t \in[a, b]$, 
- for each compact set $K \subset \mathcal{A}$, there exists a function $m_{K} \in L^{1}([a, b])$ such that $|f(t, x)| \leq m_{K}(t)$ for a.e. $t \in[a, b]$ and each $x \in K$.

We say that $z:[0, T] \rightarrow \mathbb{R}$ is a solution of problem (1)-(3), if $z$ is continuous on $[0, T]$, there exists unique $\tau \in(0, T)$ such that $\gamma(z(\tau))=\tau,\left.z\right|_{[0, \tau]}$ and $\left.z\right|_{[\tau, T]}$ have absolutely continuous first derivatives, $z$ satisfies equation (1) for a.e. $t \in[0, T]$ and fulfills conditions (2), (3).

\section{Operators}

In this section we assume that (4)-(8) are fulfilled. We introduce sets and operators corresponding to problem (1)-(3) and prove their properties which are needed for an application of the Leray-Schauder degree theory. Let us consider $K$ of (7) and define the set

$$
B_{1}=\left\{u \in C^{1}([0, T]):\|u\|_{\infty}<K,\left\|u^{\prime}\right\|_{\infty}<K / T\right\} .
$$

Lemma 1 For each $u \in \bar{B}_{1}$, there exists a unique $\tau_{u} \in(0, T)$ such that

$$
\gamma\left(u\left(\tau_{u}\right)\right)=\tau_{u}
$$

Proof Let us take an arbitrary $u \in \bar{B}_{1}$. Obviously, the constant $\tau_{u}$ is a solution of the equation

$$
\gamma(u(t))=t
$$

i.e., $\tau_{u}$ is a root of the function

$$
\sigma(t)=\gamma(u(t))-t, \quad t \in[0, T]
$$

From (8) it follows $\sigma(0)=\gamma(u(0))>0, \sigma(T)=\gamma(u(T))-T<0$. According to (8) and the definition of $B_{1}$, we get

$$
\sigma^{\prime}(t)=\gamma^{\prime}(u(t)) u^{\prime}(t)-1 \leq\left|\gamma^{\prime}(u(t))\right|\left|u^{\prime}(t)\right|-1<\frac{T}{K} \frac{K}{T}-1=0, \quad t \in(0, T) .
$$

Therefore, $\sigma$ is strictly decreasing on $[0, T]$ and hence it has exactly one root in $(0, T)$.

Now, define a functional $\mathcal{P}: \bar{B}_{1} \rightarrow(0, T)$ by

$$
\mathcal{P} u=\tau_{u},
$$

where $\tau_{u}$ fulfills (9). The next lemma provides an important result about the continuity of $\mathcal{P}$ which is fundamental for our approach.

Lemma 2 The functional $\mathcal{P}$ is continuous on $\bar{B}_{1}$.

Proof Let us consider $u_{n}, u \in \bar{B}_{1}$ for $n \in \mathbb{N}$ such that $u_{n} \rightarrow u$ in $C^{1}([0, T])$. Let us denote

$$
\sigma_{n}(t)=\gamma\left(u_{n}(t)\right)-t, \quad \sigma(t)=\gamma(u(t))-t \quad \text { for } t \in[0, T] .
$$


By Lemma $1, \sigma_{n}\left(\tau_{n}\right)=0$ and $\sigma(\tau)=0$, where $\tau_{n}=\mathcal{P} u_{n}$ and $\tau=\mathcal{P} u$, respectively. According to (8), we get $\sigma_{n}, \sigma \in C^{1}([0, T])$ for $n \in \mathbb{N}$ and

$$
\sigma_{n} \rightarrow \sigma \quad \text { in } C([0, T])
$$

We will prove that $\lim _{n \rightarrow \infty} \tau_{n}=\tau$. Let us take an arbitrary $\epsilon>0$. Since $\sigma(\tau)=0$ and $\sigma^{\prime}(\tau)<$ $0(c f .(10))$, we can find $\xi \in(\tau-\epsilon, \tau)$ and $\eta \in(\tau, \tau+\epsilon)$ such that

$$
\sigma(\xi)>0 \quad \text { and } \quad \sigma(\eta)<0
$$

From (11) it follows the existence of $n_{0} \in \mathbb{N}$ such that

$$
\sigma_{n}(\xi)>0 \text { and } \sigma_{n}(\eta)<0
$$

for each $n \geq n_{0}$. By Lemma 1 and the continuity of $\sigma_{n}$, it follows that $\tau_{n} \in(\xi, \eta) \subset(\tau-\epsilon, \tau+$ $\epsilon)$ for $n \geq n_{0}$.

Further, consider $K_{1}$ of (8) and define sets $B_{2}$ and $\Omega$ by

$$
B_{2}=\left\{v \in C^{1}([0, T]):\|v\|_{\infty}<K_{1},\left\|v^{\prime}\right\|_{\infty}<\frac{K_{1}}{T}\right\}
$$

and

$$
\Omega=B_{1} \times B_{2} \subset C^{1}([0, T]) \times C^{1}([0, T]) .
$$

Finally, define an operator $\mathcal{F}: \bar{\Omega} \rightarrow C^{1}([0, T]) \times C^{1}([0, T])$ by $\mathcal{F}(u, v)=(x, y)$, where

$$
\left\{\begin{array}{l}
x(t)=\int_{0}^{T} G(t, s) \tilde{f}(s, u(s), v(s)) \mathrm{d} s+g_{1}\left(t, \tau_{u}\right) I\left(u\left(\tau_{u}\right)\right), \\
y(t)=\int_{0}^{T} G(t, s) \tilde{f}(s, u(s), v(s)) \mathrm{d} s+g_{2}\left(t, \tau_{u}\right) I\left(u\left(\tau_{u}\right)\right),
\end{array}\right.
$$

for $t \in[0, T], \tau_{u}=\mathcal{P} u$,

$$
\begin{aligned}
& \tilde{f}(t, u(t), v(t))= \begin{cases}f(t, u(t)) & \text { for a.e. } t \in\left[0, \tau_{u}\right], \\
f(t, v(t)) & \text { for a.e. } t \in\left(\tau_{u}, T\right],\end{cases} \\
& g_{1}(t, s)=\frac{t(s-T)}{T}, \quad g_{2}(t, s)=\frac{s(t-T)}{T}, \quad s, t \in[0, T],
\end{aligned}
$$

and $G$ is the Green function of the problem $u^{\prime \prime}=0, u(0)=u(T)=0$, that is,

$$
G(t, s)= \begin{cases}g_{1}(t, s) & \text { for } 0 \leq t \leq s \leq T \\ g_{2}(t, s) & \text { for } 0 \leq s \leq t \leq T\end{cases}
$$

Lemma 3 The operator $\mathcal{F}$ is compact on $\bar{\Omega}$. 
Proof First, we will prove the continuity of the operator $\mathcal{F}$. Let us choose $\left(u_{n}, v_{n}\right),(u, v) \in \bar{\Omega}$ for $n \in \mathbb{N}$ such that

$$
\left(u_{n}, v_{n}\right) \rightarrow(u, v) \quad \text { in } C^{1}([0, T]) \times C^{1}([0, T]) .
$$

Let us denote $\tau_{n}=\mathcal{P} u_{n}, \tau=\mathcal{P} u,\left(x_{n}, y_{n}\right)=\mathcal{F}\left(u_{n}, v_{n}\right),(x, y)=\mathcal{F}(u, v)$ for each $n \in \mathbb{N}$. We will prove that $x_{n} \rightarrow x$ in $C^{1}([0, T])$. For each $t \in[0, T]$, we get by (13)-(15)

$$
\begin{aligned}
x_{n}(t)-x(t)= & \int_{0}^{\tau} G(t, s)\left[f\left(s, u_{n}(s)\right)-f(s, u(s))\right] \mathrm{d} s \\
& +\int_{\tau}^{T} G(t, s)\left[f\left(s, v_{n}(s)\right)-f(s, v(s))\right] \mathrm{d} s \\
& +\int_{\tau}^{\tau_{n}} G(t, s)\left[f\left(s, u_{n}(s)\right)-f\left(s, v_{n}(s)\right)\right] \mathrm{d} s \\
& +g_{1}\left(t, \tau_{n}\right) I\left(u_{n}\left(\tau_{n}\right)\right)-g_{1}(t, \tau) I(u(\tau))
\end{aligned}
$$

and

$$
\begin{aligned}
x_{n}^{\prime}(t)-x^{\prime}(t)= & \int_{0}^{\tau} \frac{\partial G}{\partial t}(t, s)\left[f\left(s, u_{n}(s)\right)-f(s, u(s))\right] \mathrm{d} s \\
& +\int_{\tau}^{T} \frac{\partial G}{\partial t}(t, s)\left[f\left(s, v_{n}(s)\right)-f(s, v(s))\right] \mathrm{d} s \\
& +\int_{\tau}^{\tau_{n}} \frac{\partial G}{\partial t}(t, s)\left[f\left(s, u_{n}(s)\right)-f\left(s, v_{n}(s)\right)\right] \mathrm{d} s \\
& +\frac{\partial g_{1}}{\partial t}\left(t, \tau_{n}\right) I\left(u_{n}\left(\tau_{n}\right)\right)-\frac{\partial g_{1}}{\partial t}(t, \tau) I(u(\tau)) .
\end{aligned}
$$

Since

$$
|G(t, s)| \leq T, \quad\left|\frac{\partial G}{\partial t}(t, s)\right| \leq 1 \quad \text { for } t, s \in[0, T], t \neq s,
$$

we get

$$
\begin{aligned}
\left\|x_{n}-x\right\|_{1} \leq & (T+1) \int_{0}^{T}\left|f\left(s, u_{n}(s)\right)-f(s, u(s))\right| \mathrm{d} s \\
& +(T+1) \int_{0}^{T}\left|f\left(s, v_{n}(s)\right)-f(s, v(s))\right| \mathrm{d} s \\
& +(T+1) \int_{\tau}^{\tau_{n}}\left|f\left(s, u_{n}(s)\right)-f\left(s, v_{n}(s)\right)\right| \mathrm{d} s \\
& +\max _{t \in[0, T]}\left|g_{1}\left(t, \tau_{n}\right) I\left(u_{n}\left(\tau_{n}\right)\right)-g_{1}(t, \tau) I(u(\tau))\right| \\
& +\max _{t \in[0, T]}\left|\frac{\partial g_{1}}{\partial t}\left(t, \tau_{n}\right) I\left(u_{n}\left(\tau_{n}\right)\right)-\frac{\partial g_{1}}{\partial t}(t, \tau) I(u(\tau))\right| .
\end{aligned}
$$

By (16), there exists a compact set $K \subset \mathbb{R}$ such that $u_{n}(t), v_{n}(t) \in K$ for each $t \in[0, T]$ and $n \in \mathbb{N}$. Consequently, by (4), there exists $m_{K} \in L^{1}([0, T])$ such that

$$
\left|f\left(t, u_{n}(t)\right)\right| \leq m_{K}(t), \quad\left|f\left(t, v_{n}(t)\right)\right| \leq m_{K}(t)
$$


for a.e. $t \in[0, T]$ and all $n \in \mathbb{N}$. Since

$$
\lim _{n \rightarrow \infty} f\left(t, u_{n}(t)\right)=f(t, u(t)), \quad \lim _{n \rightarrow \infty} f\left(t, v_{n}(t)\right)=f(t, v(t))
$$

for a.e. $t \in[0, T]$, then due to the Lebesgue dominated convergence theorem, it follows that

$$
\int_{0}^{T}\left|f\left(s, u_{n}(s)\right)-f(s, u(s))\right| \mathrm{d} s \rightarrow 0 \quad \text { and } \quad \int_{0}^{T}\left|f\left(s, v_{n}(s)\right)-f(s, v(s))\right| \mathrm{d} s \rightarrow 0
$$

as $n \rightarrow \infty$. Since $\lim _{n \rightarrow \infty} \tau_{n}=\tau$, the absolute continuity of the Lebesgue integral yields

$$
\lim _{n \rightarrow \infty}\left|\int_{\tau}^{\tau_{n}}\right| f\left(s, u_{n}(s)\right)-f\left(s, v_{n}(s)\right)|\mathrm{d} s| \leq 2 \lim _{n \rightarrow \infty}\left|\int_{\tau}^{\tau_{n}} m_{K}(s) \mathrm{d} s\right|=0 .
$$

Further, we have for $g_{1}$ the inequality

$$
\begin{aligned}
& \left|g_{1}\left(t, \tau_{n}\right) I\left(u_{n}\left(\tau_{n}\right)\right)-g_{1}(t, \tau) I(u(\tau))\right| \\
& \quad \leq T\left|I\left(u_{n}\left(\tau_{n}\right)\right)-I\left(u\left(\tau_{n}\right)\right)\right|+\left|g_{1}\left(t, \tau_{n}\right) I\left(u\left(\tau_{n}\right)\right)-g_{1}(t, \tau) I(u(\tau))\right|
\end{aligned}
$$

for each $t \in[0, T]$ and the same is true for $\frac{\partial g_{1}}{\partial t}$. The continuity of $g_{1}$, $\frac{\partial g_{1}}{\partial t}$ and $I$ imply that

$$
g_{1}\left(t, \tau_{n}\right) I\left(u_{n}\left(\tau_{n}\right)\right) \rightarrow g_{1}(t, \tau) I(u(\tau)), \quad \frac{\partial g_{1}}{\partial t}\left(t, \tau_{n}\right) I\left(u_{n}\left(\tau_{n}\right)\right) \rightarrow \frac{\partial g_{1}}{\partial t}(t, \tau) I(u(\tau))
$$

as $n \rightarrow \infty$ uniformly w.r.t. $t \in[0, T]$. Therefore, $x_{n}$ converges to $x$ in $C^{1}([0, T])$. Similar arguments can be applied to the sequence $\left\{y_{n}\right\}_{n=1}^{\infty}$.

Now we will prove that $\mathcal{F}(\bar{\Omega})$ is relatively compact. The boundedness of $\bar{\Omega}$ implies the existence of $M>0$ and $m \in L^{1}([0, T])$ such that for all $(u, v) \in \bar{\Omega}$,

$$
|I(u(t))| \leq M \quad \text { for all } t \in[0, T]
$$

and

$$
|\tilde{f}(t, u(t), v(t))| \leq m(t) \quad \text { for a.e. } t \in[0, T] .
$$

Therefore, by (13), we get

$$
\begin{aligned}
& |x(t)|+\left|x^{\prime}(t)\right| \leq(T+1)\left(\int_{0}^{T} m(s) \mathrm{d} s+M\right)=: M_{1}, \quad t \in[0, T], \\
& |y(t)|+\left|y^{\prime}(t)\right| \leq M_{1}, \quad t \in[0, T] .
\end{aligned}
$$

We have proved that the set $\mathcal{F}(\bar{\Omega})$ is bounded in $C^{1}([0, T]) \times C^{1}([0, T])$. We now show that the set $\left\{\left(x^{\prime}, y^{\prime}\right):(x, y) \in \mathcal{F}(\bar{\Omega})\right\}$ is equicontinuous on $[0, T]$. For a.e. $t \in[0, T]$ and all $(x, y) \in \mathcal{F}(\bar{\Omega})$, we have

$$
\left|x^{\prime \prime}(t)\right| \leq m(t), \quad\left|y^{\prime \prime}(t)\right| \leq m(t) \quad \text { for a.e. } t \in[0, T] .
$$


As a result, for each $\epsilon>0$, there exists $\delta>0$ such that for each $t_{1}, t_{2} \in[0, T]$ satisfying $\left|t_{1}-t_{2}\right|<\delta$, the inequality

$$
\left|x^{\prime}\left(t_{1}\right)-x^{\prime}\left(t_{2}\right)\right|+\left|y^{\prime}\left(t_{1}\right)-y^{\prime}\left(t_{2}\right)\right| \leq 2\left|\int_{t_{2}}^{t_{1}} m(t) \mathrm{d} t\right|<\epsilon
$$

holds for all $(x, y) \in \mathcal{F}(\bar{\Omega})$. Consequently, $\mathcal{F}(\bar{\Omega})$ is relatively compact in $C^{1}([0, T]) \times$ $C^{1}([0, T])$ by the Arzelà-Ascoli theorem.

Lemma 4 Let $(u, v) \in \bar{\Omega}$ be a fixed point of $\mathcal{F}$. Then the function

$$
z(t)= \begin{cases}u(t), & t \in\left[0, \tau_{u}\right] \\ v(t), & t \in\left(\tau_{u}, T\right]\end{cases}
$$

is a solution of problem (1)-(3).

Proof Let $(u, v) \in \bar{\Omega}$ be such that $(u, v)=\mathcal{F}(u, v)$, that is,

$$
\left\{\begin{array}{l}
u(t)=\int_{0}^{T} G(t, s) \tilde{f}(s, u(s), v(s)) \mathrm{d} s+g_{1}\left(t, \tau_{u}\right) I\left(u\left(\tau_{u}\right)\right), \\
v(t)=\int_{0}^{T} G(t, s) \tilde{f}(s, u(s), v(s)) \mathrm{d} s+g_{2}\left(t, \tau_{u}\right) I\left(u\left(\tau_{u}\right)\right),
\end{array}\right.
$$

$t \in[0, T], \tau_{u}=\mathcal{P} u$.

Let us consider the function $z$ defined in (17). Hence, $z(0)=u(0)=0, z(T)=v(T)=0$,

$$
z\left(\tau_{u}\right)=u\left(\tau_{u}\right)=v\left(\tau_{u}\right)=z\left(\tau_{u}+\right),
$$

and by Lemma 1 ,

$$
\gamma\left(z\left(\tau_{u}\right)\right)=\tau_{u}
$$

In addition, by (17), $\tau_{u}$ is a unique point in $\left(0, \tau_{u}\right]$ satisfying (20). Put $\sigma(t)=\gamma(v(t))-t$, $t \in\left[\tau_{u}, T\right]$. Due to (19) and (20), we get $\sigma\left(\tau_{u}\right)=0$. Further,

$$
\begin{aligned}
\sigma^{\prime}(t) & =\gamma^{\prime}(v(t)) v^{\prime}(t)-1 \leq\left|\gamma^{\prime}(v(t))\right|\left|v^{\prime}(t)\right|-1 \\
& <\frac{T}{K_{1}} \frac{K_{1}}{T}-1=0 \quad \text { for } t \in\left(\tau_{u}, T\right) .
\end{aligned}
$$

Therefore, $\sigma$ is strictly decreasing on $\left[\tau_{u}, T\right]$, which yields $\sigma(t)<0$ for $t \in\left(\tau_{u}, T\right]$. Consequently, $\tau_{u}$ is a unique point in $(0, T)$ satisfying $(20)$.

Further, we get

$$
\begin{aligned}
& u^{\prime}(t)=\int_{0}^{T} \frac{\partial G}{\partial t}(t, s) \tilde{f}(s, u(s), v(s)) \mathrm{d} s+\frac{\partial g_{1}}{\partial t}\left(t, \tau_{u}\right) I\left(u\left(\tau_{u}\right)\right), \\
& v^{\prime}(t)=\int_{0}^{T} \frac{\partial G}{\partial t}(t, s) \tilde{f}(s, u(s), v(s)) \mathrm{d} s+\frac{\partial g_{2}}{\partial t}\left(t, \tau_{u}\right) I\left(u\left(\tau_{u}\right)\right),
\end{aligned}
$$


$t \in[0, T]$, and by virtue of (14),

$$
\begin{array}{ll}
u^{\prime \prime}(t)=\tilde{f}(t, u(t), v(t))=f(t, u(t)) & \text { for a.e. } t \in\left[0, \tau_{u}\right), \\
v^{\prime \prime}(t)=\tilde{f}(t, u(t), v(t))=f(t, v(t)) & \text { for a.e. } t \in\left(\tau_{u}, T\right] .
\end{array}
$$

Therefore,

$$
z^{\prime \prime}(t)=f(t, z(t)) \quad \text { for a.e. } t \in[0, T] .
$$

Finally,

$$
\begin{aligned}
& z^{\prime}\left(\tau_{u}+\right)=v^{\prime}\left(\tau_{u}\right)=\int_{0}^{T} \frac{\partial G}{\partial t}\left(\tau_{u}+, s\right) \tilde{f}(s, u(s), v(s)) \mathrm{d} s+\frac{\partial g_{2}}{\partial t}\left(\tau_{u}, \tau_{u}\right) I\left(u\left(\tau_{u}\right)\right), \\
& z^{\prime}\left(\tau_{u^{-}}\right)=u^{\prime}\left(\tau_{u}\right)=\int_{0}^{T} \frac{\partial G}{\partial t}\left(\tau_{u^{-}}, s\right) \tilde{f}(s, u(s), v(s)) \mathrm{d} s+\frac{\partial g_{1}}{\partial t}\left(\tau_{u}, \tau_{u}\right) I\left(u\left(\tau_{u}\right)\right) .
\end{aligned}
$$

Since

$$
\frac{\partial G}{\partial t}\left(\tau_{u^{+}}, s\right)=\frac{\partial G}{\partial t}\left(\tau_{u^{-}}, s\right) \quad \text { for } s \in[0, T], s \neq \tau_{u},
$$

we have

$$
\begin{aligned}
z^{\prime}\left(\tau_{u^{+}}\right)-z^{\prime}\left(\tau_{u^{-}}\right) & =\frac{\partial g_{2}}{\partial t}\left(\tau_{u}, \tau_{u}\right) I\left(u\left(\tau_{u}\right)\right)-\frac{\partial g_{1}}{\partial t}\left(\tau_{u}, \tau_{u}\right) I\left(u\left(\tau_{u}\right)\right) \\
& =\frac{\tau_{u}}{T} I\left(u\left(\tau_{u}\right)\right)-\frac{\tau_{u}-T}{T} I\left(u\left(\tau_{u}\right)\right)=I\left(u\left(\tau_{u}\right)\right)=I\left(z\left(\tau_{u}\right)\right) .
\end{aligned}
$$

\section{Main result}

Here, using the Leray-Schauder degree theory, we prove our main result about the solvability of problem (1)-(3). To this end, we will need the following lemma on a priori estimates.

Lemma 5 Assume (4)-(8). Then for any $\lambda \in[0,1]$ and any solution $(u, v)$ of the equation

$$
(u, v)=\lambda \mathcal{F}(u, v),
$$

the implication

$$
(u, v) \in \bar{\Omega} \quad \Longrightarrow \quad(u, v) \in \Omega
$$

holds.

Proof Let us choose $\lambda \in(0,1]$ and let $(u, v) \in \bar{\Omega}$ satisfy (21), i.e.,

$$
\left\{\begin{array}{l}
u(t)=\lambda\left(\int_{0}^{T} G(t, s) \tilde{f}(s, u(s), v(s)) \mathrm{d} s+g_{1}\left(t, \tau_{u}\right) I\left(u\left(\tau_{u}\right)\right)\right), \\
v(t)=\lambda\left(\int_{0}^{T} G(t, s) \tilde{f}(s, u(s), v(s)) \mathrm{d} s+g_{2}\left(t, \tau_{u}\right) I\left(u\left(\tau_{u}\right)\right)\right)
\end{array}\right.
$$

for $t \in[0, T]$. Then

$$
\left\{\begin{array}{l}
u^{\prime}(t)=\lambda\left(\int_{0}^{T} \frac{\partial G}{\partial t}(t, s) \tilde{f}(s, u(s), v(s)) \mathrm{d} s+\frac{\partial g_{1}}{\partial t}\left(t, \tau_{u}\right) I\left(u\left(\tau_{u}\right)\right)\right), \\
v^{\prime}(t)=\lambda\left(\int_{0}^{T} \frac{\partial G}{\partial t}(t, s) \tilde{f}(s, u(s), v(s)) \mathrm{d} s+\frac{\partial g_{2}}{\partial t}\left(t, \tau_{u}\right) I\left(u\left(\tau_{u}\right)\right)\right)
\end{array}\right.
$$


$t \in[0, T]$. Since $(u, v) \in \bar{\Omega}$, it follows that $(u, v) \in \bar{B}_{1} \times \bar{B}_{2}$ and therefore $\left\|u^{\prime}\right\|_{\infty} \leq K / T$, $\|u\|_{\infty} \leq K,\left\|v^{\prime}\right\|_{\infty} \leq K_{1} / T$ and $\|v\|_{\infty} \leq K_{1}$. There are two possibilities as follows.

Case A. Let $\left\|u^{\prime}\right\|_{\infty}<K / T$. Then $\|u\|_{\infty}<K$ and from (15) and (24), it follows

$$
\begin{aligned}
v^{\prime}(t) & =u^{\prime}(t)+\lambda\left(\frac{\tau_{u}}{T}-\frac{\tau_{u}-T}{T}\right) I\left(u\left(\tau_{u}\right)\right) \\
& =u^{\prime}(t)+\lambda I\left(u\left(\tau_{u}\right)\right), \quad t \in(0, T),
\end{aligned}
$$

which implies, due to (6) and (8),

$$
\left\|v^{\prime}\right\|_{\infty} \leq\left\|u^{\prime}\right\|_{\infty}+J\left(\|u\|_{\infty}\right)<\frac{K_{1}}{T}
$$

Then $\|v\|_{\infty}<K_{1}$, which yields $(u, v) \in \Omega$.

Case B. Let $\left\|u^{\prime}\right\|_{\infty}=K / T$. From (24), (14), (6), (5) and (8), it follows

$$
\begin{aligned}
\left\|u^{\prime}\right\| & \leq \int_{0}^{\tau_{u}}|f(s, u(s))| \mathrm{d} s+\int_{\tau_{u}}^{T}|f(s, v(s))| \mathrm{d} s+J(K) \\
& \leq \int_{0}^{\tau_{u}} h(s, K) \mathrm{d} s+\int_{\tau_{u}}^{T} h\left(s, K_{1}\right) \mathrm{d} s+J(K) \\
& \leq \int_{0}^{T} h\left(s, K_{1}\right) \mathrm{d} s+J(K) .
\end{aligned}
$$

This inequality together with (7) implies

$$
1 \leq \frac{T}{K}\left[\int_{0}^{T} h\left(s, K_{1}\right) \mathrm{d} s+J(K)\right]<T \min \left\{1, \frac{1}{T}\right\}=\min \{T, 1\},
$$

which is a contradiction.

For $\lambda=0$, the solution of $(21)$ is $(u, v)=(0,0)$, and it clearly belongs to $\Omega$.

Theorem 6 Assume (4)-(8). Then the operator $\mathcal{F}$ has a fixed point in $\Omega$.

Proof According to Lemma 5, the operator $I-\lambda \mathcal{F}:[0,1] \times \bar{\Omega} \rightarrow\left(C^{1}([0, T])\right)^{2}$ is a homotopy. Therefore,

$$
\operatorname{deg}(I-\mathcal{F}, \Omega)=\operatorname{deg}(I, \Omega)=1,
$$

and consequently the equation

$$
(I-\mathcal{F})(u, v)=0
$$

has a solution in $\Omega$. This solution is a fixed point of the operator $\mathcal{F}$.

Theorem 7 Assume (4)-(8). Then problem (1)-(3) has a solution $z$ such that

$$
\|z\|_{\infty}<K_{1}, \quad\left\|z^{\prime}\right\|_{\infty}<\frac{K_{1}}{T}
$$


Proof From Theorem 6 it follows that there exists a fixed point $(u, v) \in \Omega$ of the operator $\mathcal{F}$. Lemma 4 yields that the function $z$ defined in (17) (with $\tau_{u}=\mathcal{P} u$ ) is a solution of problem (1)-(3). Estimates (25) follow from (17) and from the definitions of $\Omega$ and $K_{1}$ (cf. (12) and (8)).

Remark 8 Let us note that assumption (7) follows from the condition

$$
\liminf _{x \rightarrow \infty} \frac{1}{x}\left[\int_{0}^{T} h(s, x+T J(x)) \mathrm{d} s+J(x)\right]<\min \left\{1, \frac{1}{T}\right\} .
$$

\section{Examples}

In this section we demonstrate that Theorem 7 can be applied to sublinear, linear and superlinear problems.

Example 9 (Sublinear problem) Let us consider problem (1)-(3) with

$$
T=1, \quad f(t, x)=t^{2}-|x|^{\alpha} \operatorname{sgn} x, \quad I(x)=|x|^{\beta} \operatorname{sgn} x, \quad \alpha, \beta \in(0,1)
$$

that is, $f$ and $I$ are sublinear in $x$. Then assumptions (5) and (6) are valid for

$$
\begin{aligned}
& h(t, x)=t^{2}+x^{\alpha}, \quad t \in[0,1], x>0, \\
& J(x)=x^{\beta}, \quad x>0 .
\end{aligned}
$$

Since

$$
\lim _{x \rightarrow \infty} \frac{1}{x}\left[\int_{0}^{1} h(s, x+J(x)) \mathrm{d} s+J(x)\right]=\lim _{x \rightarrow \infty} \frac{1}{x}\left[\frac{1}{3}+\left(x+x^{\beta}\right)^{\alpha}+x^{\beta}\right]=0,
$$

Remark 8 yields that condition (7) is satisfied for any sufficiently large $K$. In particular, let us put

$$
\alpha=\beta=\frac{1}{2} .
$$

If we choose $K=10$, we see that (7) holds. Then by (8), we have

$$
K_{1}=10+\sqrt{10} .
$$

For instance, if we choose $c \in\left(0,1 /\left(2 K_{1}^{2}\right)\right)$ and put

$$
\gamma(x)=c x^{2}+\frac{1}{2}, \quad x \in \mathbb{R}
$$

or if we choose $c \in(0,1 / 2), n>c K_{1}$ and put

$$
\gamma(x)=c \sin \frac{x}{n}+\frac{1}{2}, \quad x \in \mathbb{R}
$$

we can check that conditions (8) are satisfied in both cases. Therefore, by Theorem 7, the corresponding problem (1)-(3) has at least one solution. 
Note that (27) shows that $\gamma$ need not be monotonous.

Example 10 (Linear problem) Let us consider problem (1)-(3) with $f$ and $I$ having the linear behavior in $x$ and put

$$
T=1, \quad f(t, x)=a\left(t^{\alpha}-x\right), \quad I(x)=b x, \quad a, b \in \mathbb{R}, \alpha>0 .
$$

Then assumptions (5) and (6) are valid for

$$
\begin{aligned}
& h(t, x)=|a|\left(t^{\alpha}+x\right), \quad t \in[0,1], x>0, \\
& J(x)=|b| x, \quad x>0 .
\end{aligned}
$$

Since

$$
\begin{aligned}
\lim _{x \rightarrow \infty} & \frac{1}{x}\left[\int_{0}^{1} h(s, x+J(x)) \mathrm{d} s+J(x)\right] \\
= & \lim _{x \rightarrow \infty} \frac{1}{x}\left[|a|\left(\frac{1}{\alpha+1}+x(1+|b|)\right)+x|b|\right]=|a|(1+|b|)+|b|,
\end{aligned}
$$

Theorem 7 can be applied, due to Remark 8, under the additional assumption

$$
|a|<\frac{1-|b|}{1+|b|}
$$

If (28) holds, then for any sufficiently large $K$, condition (7) is satisfied. By (8), we have $K_{1}=K(1+|b|)$, and problem (1)-(3) has a solution for any $\gamma$ satisfying (8). Consequently, if $\gamma$ is given by (26) or (27), problem (1)-(3) is solvable.

Example 11 (Superlinear problem) Let us consider problem (1)-(3) with $f$ and $I$ superlinear in $x$. Put, for example,

$$
T=1, \quad f(t, x)=c_{1} t^{3}+c_{2} x^{3}, \quad I(x)=\frac{1}{2} x^{2}, \quad c_{1}, c_{2} \in \mathbb{R}
$$

Then assumptions (5) and (6) are valid for

$$
\begin{aligned}
& h(t, x)=\left|c_{1}\right| t^{3}+\left|c_{2}\right| x^{3}, \quad t \in[0,1], x>0, \\
& J(x)=\frac{1}{2} x^{2}, \quad x>0 .
\end{aligned}
$$

It holds

$$
\frac{1}{x}\left[\int_{0}^{1} h(s, x+J(x)) \mathrm{d} s+J(x)\right]=\frac{1}{x}\left[\frac{\left|c_{1}\right|}{4}+\left|c_{2}\right|\left(x+\frac{1}{2} x^{2}\right)^{3}+\frac{1}{2} x^{2}\right] .
$$

By virtue of (7), Theorem 7 can be applied provided there exists $K>0$ such that

$$
\frac{\left|c_{1}\right|}{4}+\left|c_{2}\right|\left(K+\frac{1}{2} K^{2}\right)^{3}+\frac{1}{2} K^{2}<K
$$


Let us search $K$ in the interval $(0,1)$. Then $K^{3}<K^{2}<K$ and it holds

$$
\left|c_{2}\right|\left(K+\frac{1}{2} K^{2}\right)^{3}+\frac{1}{2} K^{2}<\left|c_{2}\right|\left(\frac{3}{2} K\right)^{3}+\frac{1}{2} K^{2}<\left(\frac{27}{8}\left|c_{2}\right|+\frac{1}{2}\right) K^{2} .
$$

Consequently, each $K \in(0,1)$ fulfilling the equation

$$
\left(\frac{27}{8}\left|c_{2}\right|+\frac{1}{2}\right) K^{2}-K+\frac{\left|c_{1}\right|}{4}=0
$$

satisfies (30) as well. Put, for example, $c_{1}=1, c_{2}=-4 / 27$. Then we get that for $K=1 / 2$ inequality (30) holds. Consequently, (8) gives $K_{1}=5 / 8$ and the corresponding problem (1)-(3) is solvable for any $\gamma$ satisfying (8). In particular, $\gamma$ given by (26) or (27) can be considered in this case as well.

\section{Competing interests}

The authors declare that they have no competing interests.

\section{Authors' contributions}

Both authors contributed equally to the manuscript and read and approved the final manuscript.

\section{Acknowledgements}

Dedicated to Jean Mawhin on the occasion of his 70th birthday.

The authors would like to thank the anonymous referees for their valuable comments and suggestions. This work was supported by the grant Matematické modely a struktury, PrF_2012_017.

Received: 24 October 2012 Accepted: 15 January 2013 Published: 11 February 2013

\section{References}

1. Bainov, D, Simeonov, P: Impulsive Differential Equations: Periodic Solutions and Applications. Pitman Monographs and Surveys in Pure and Applied Mathematics, vol. 66. Longman, Essex (1993)

2. Lakshmikantham, V, Bainov, DD, Simeonov, PS: Theory of Impulsive Differential Equations. World Scientific, Singapore (1989)

3. Samoilenko, AM, Perestyuk, NA: Impulsive Differential Equations. World Scientific, Singapore (1995)

4. Afonso, SM, Bonotto, EM, Federson, M, Schwabik, Š: Discontinuous local semiflows for Kurzweil equations leading to LaSalle's invariance principle for differential systems with impulses at variable times. J. Differ. Equ. 250, 2969-3001 (2011)

5. Benchohra, M, Henderson, J, Ntouyas, SK, Ouahab, A: Impulsive functional differential equations with variable times. Comput. Math. Appl. 47, 1659-1665 (2004)

6. Domoshnitsky, A, Drakhlin, M, Litsyn, E: Nonoscillation and positivity of solutions to first order state-dependent differential equations with impulses in variable moments. J. Differ. Equ. 228, 39-48 (2006)

7. Frigon, M, O’Regan, D: Impulsive differential equations with variable times. Nonlinear Anal. 26, 1913-1922 (1996)

8. Kaul, S, Lakshmikantham, V, Leela, S: Extremal solutions, comparison principle and stability criteria for impulsive differential equations with variable times. Nonlinear Anal. 22, 1263-1270 (1994)

9. Liu, L, Sun, J: Existence of periodic solution of a harvested system with impulses at variable times. Phys. Lett. A 360 , 105-108 (2006)

10. Qi, J, Fu, X: Existence of limit cycles of impulsive differential equations with impulses at variable times. Nonlinear Anal. 44, 345-353 (2001)

11. Bajo, I, Liz, E: Periodic boundary value problem for first order differential equations with impulses at variable times. J. Math. Anal. Appl. 204, 65-73 (1996)

12. Belley, J, Virgilio, M: Periodic Duffing delay equations with state dependent impulses. J. Math. Anal. Appl. 306, 646-662 (2005)

13. Belley, J, Virgilio, M: Periodic Liénard-type delay equations with state-dependent impulses. Nonlinear Anal. 64 568-589 (2006)

14. Frigon, M, O'Regan, D: First order impulsive initial and periodic problems with variable moments. J. Math. Anal. Appl. 233, 730-739 (1999)

15. Yong, L, Fuzhong, C, Zhanghua, L: Boundary value problems for impulsive differential equations. Nonlinear Anal. TMA 29, 1253-1264 (1997)

16. Benchohra, M, Graef, JR, Ntouyas, SK, Ouahab, A: Upper and lower solutions method for impulsive differential inclusions with nonlinear boundary conditions and variable times. Dyn. Contin. Discrete Impuls. Syst. 12, 383-396 (2005)

17. Frigon, M, O'Regan, D: Second order Sturm-Liouville BVP's with impulses at variable times. Dyn. Contin. Discrete Impuls. Syst. 8, 149-159 (2001) 
doi:10.1186/1687-2770-2013-22

Cite this article as: Rachünová and Tomeček: A new approach to BVPs with state-dependent impulses. Boundary Value Problems 2013 2013:22.

Submit your manuscript to a SpringerOpen ${ }^{\circ}$ journal and benefit from:

- Convenient online submission

- Rigorous peer review

- Immediate publication on acceptance

Open access: articles freely available online

- High visibility within the field

- Retaining the copyright to your article

Submit your next manuscript at $\boldsymbol{s p r i n g e r o p e n . c o m ~}$ 